\title{
The calculation analysis of the geometric parameters for shock separation during threshing
}

\author{
Elena Fisunova ${ }^{1}$, Tatyana Lavrenova ${ }^{1, *}$, and Olga Baryshnikova $^{1}$ \\ ${ }^{1}$ FGBOU VO «Don State Technical University» (DSTU), 344000, Rostov-on-Don, Russian \\ Federation
}

\begin{abstract}
This analysis was made on the basis of the developed methodology for the geometric parameters calculating of the working bodies, which allows changing their sizes. The described algorithm and the program developed on the basis of it made it possible to calculate the position of the grains and the threshing elements depending on time with the ability to change the time interval within certain limits. Find collision parameters for various laws of grain motion and peripheral speeds of the drum, assuming that the mass of the threshing element is infinitely large compared to the mass of the grain.
\end{abstract}

\section{Purpose}

Develop an algorithm that describes the interaction of free grains with the threshing drum and concave, which allows you to establish the influence of the geometric parameters of grain reflective surfaces on the selection of grain, depending on time.

\section{Reaching of the goal}

The ability to calculate the position of grains and threshering elements depending on the time with the possibility of changing the time interval within certain limits.

When designing a combine harvester of regional significance, in order to eliminate such drawbacks as losses, often exceeding permissible norms under the influence of various natural factors, it is necessary to change the size of the working bodies. The quality of the combine harvester depends on the operation of the threshing apparatus, affecting the function of other systems.

The threshing process is carried out by whip striking on the free ears at the inlet, by impact on the plant mass in the middle part of the threshing channel and by grinding on the outlet of the threshing space. Impacts on threshing objects occur in different ways, depending on the type of object. This is a blow to the free ear, a blow to the ear or its fragment through the straw mass and other loads of grain connections in an unmilted ear or in its part.

\footnotetext{
*Corresponding author: bys_ka87@mail.ru
} 
Virtually absent scientifically based methods for rational parameter calculating of concave for the threshing apparatus with a larger diameter drum allowed to create it on the basis of experimental studies $[1,2,3]$ which showed that the stalks move along the concave with a thin layer without creating insurmountable obstacles for moving high-speed grains. After a number of assumptions the movement of grains in the threshing space is represented as a set of consecutive collisions with the threshing drum whips and concave straps. Separation in this case is considered to be shock. We excluded the centrifugal component of the force factors from the model it allowed us to simplify the separating action modeling of the threshing apparatus.

The algorithm, describing the interaction of free grains with the threshing drum and concave, allows us to establish the influence of the geometric parameters of their reflective surfaces on the selection of grain depending on time. The front surfaces of the whips and the thrashing elements, the planes of the concave strips, facing the drum are assumed to be reflective.

This model allows you to change the parameters: the depth of interfacial space, the angle of inclination and the peripheral speed of the threshing elements; the distance between the whips and the concave strips, the pitch, width, length and angle of inclination of the straps. The moving grains from the window of the inclined chamber are subjected to blows by the whips, after which, depending on the values of the listed parameters, they are sifted through the concave, or go outside the output threshing gap. At the same time, after a collision with a whip, the grain can be sifted through the concave, without touching the straps, or reflected from the slat back to the drum, after which it can be hit again by the blow of the scourge. Grain can penetrate into the inside of the drum, flying through the interfacial space due to high speed and with a corresponding angle of reflection from the concave, can go beyond the working zone of the threshing space through the output gap. At repeated collision of grain with a whip, the cycle is repeated with different probability of the result of separation. In our work, we consider grains in the form of material points that do not interact with each other.

The described algorithm and the program developed on its basis allowed to solve a number of tasks:

1. To calculate the position of the grains and threshing elements depending on the time with the ability to change the time interval within certain limits.

2. Find the collision parameters for different laws of grain motion and peripheral drum speeds, assuming that the mass of the threshing element is infinitely large compared to the mass of the grain.

The working elements in this case are the threshing elements of the threshing drum and concave straps. The calculation of the parameters of the reflection spares is performed taking into account the energy loss during the impact. The program is written in TurboPascal. A generalized block diagram of the algorithm is presented in Fig. 1.

Information is entered (block 2): the initial positions of the threshing elements and the peripheral speed of the drum; sizes and positions of the concave straps; the initial position, direction and speed of movement of the grains within the exit window of the inclined chamber. New positions of grains and whips (blocks 4,6) are calculated taking into account the time increment (block 3). A comparison is made of the current distance of the grain from the center of the threshing drum with its radius (blocks 7, 10, 24). If the grain is outside the threshing drum, then its index $(\mathrm{P})$ is assigned the appropriate value. The same algorithm is used in other cases (blocks 13,27, 28), but in comparison with the maximum and minimum radii of concave strap placement.

The time of the collision of grain with a whip (block 11) is found by the half division method. The use of the method is complicated due to the presence of an extremum of the function under study, which causes the need of its finding in order to clarify the limits of 
the initial interval. The collision time is calculated on the basis of their speeds and direction of movement, taking into account that the collision point is located on the nearest strap to the grain.

During the development of the algorithm, the following characteristic zones of the threshing apparatus were identified: the interfacial space, the interplanar space, the threshing gap, and the space outside the threshing apparatus, when ingested into which the grain is considered to be sieved or lost. The location of the grain in the threshing apparatus is characterized by the parameter $\mathrm{P}$. The value of $\mathrm{P}=0$ indicates that the grain is in the threshing gap, $\mathrm{P}=1$ in the interfacial space, $\mathrm{P}=2$ in the interplanar space. The fractional part of the parameter $\mathrm{P}$ indicates that the grain in this zone has already been in contact with some element of the structure, and other collisions will not occur before the grain leaves the zone.

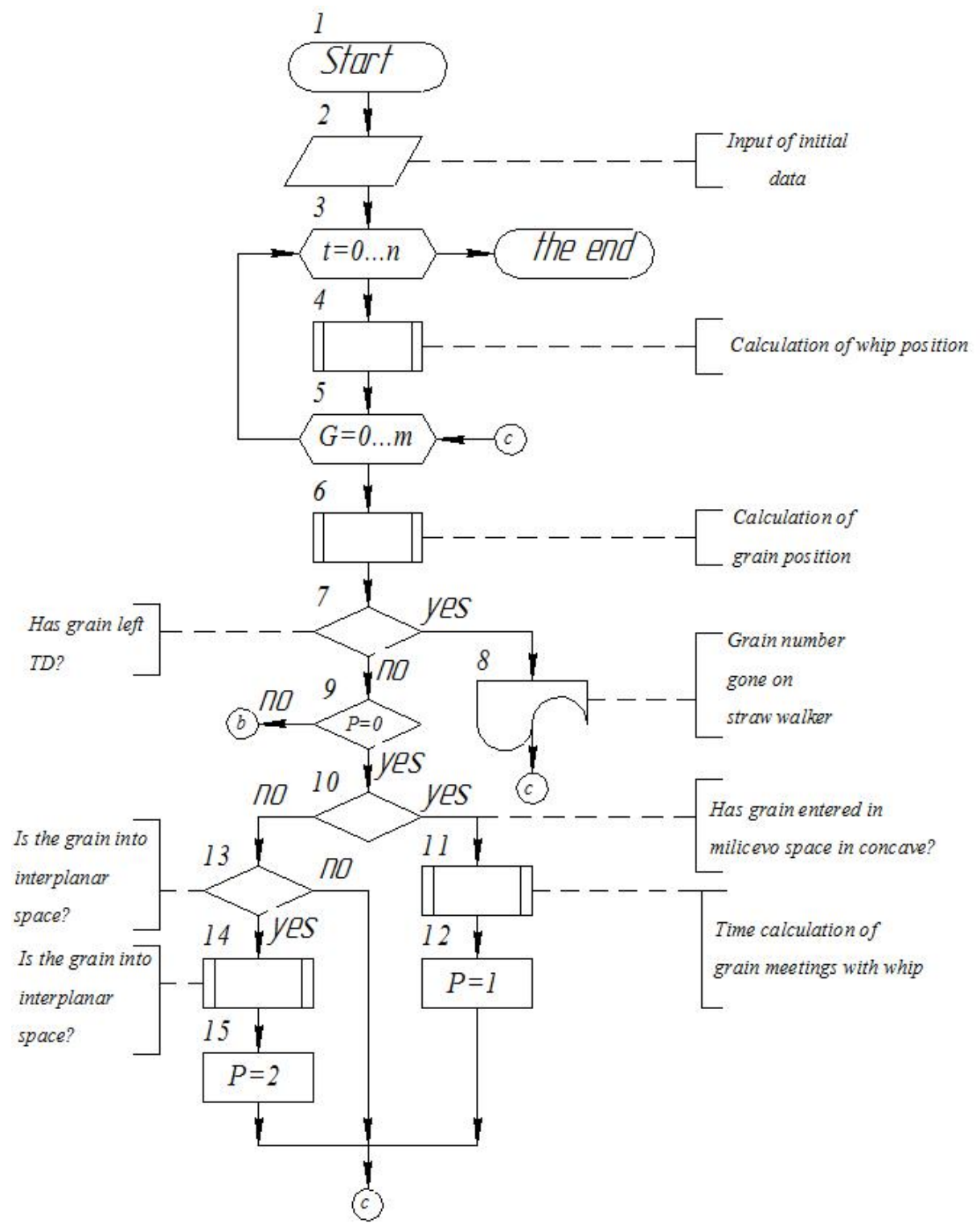




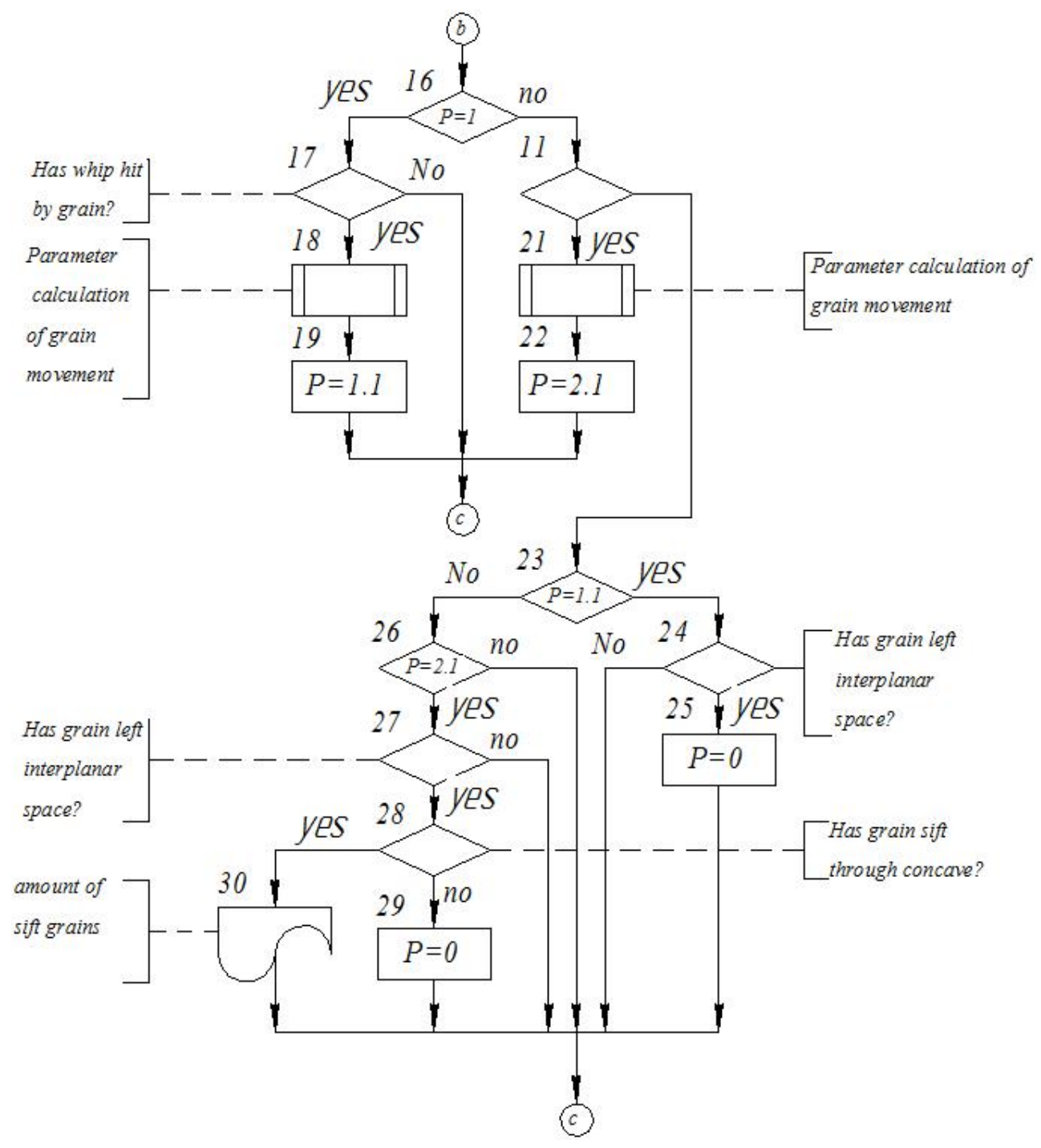

Fig. 1. The block diagram of the algorithm for parameter calculating of shock separation during threshing

The process modeled in this way and its results are displayed on the monitor screen.

\section{Conclusions}

The proposed algorithm can be used

1. At the design stage for the analysis of the separation process in various threshing devices beater of combine harvesters.

2. To find the rational parameters of the working surfaces of the threshing-separating tract based on imitation experiments. 


\section{References}

1. Antipin V.G., Korobitsin V.M. On the movement of the threshing culture by the concave // Mechanization and electrification of socialist agriculture, - (1979, - N8. - pp. 7-9).

2. Klyonin N.N. The study of threshing and separation of grain. Dis, Dr. tech. Sciences, (M. 1977 - 424).

3. Shirin V.F. Investigation of the parameters of the concave with the threshing of grain by the bil drum. Dis, Cand. tech. Sciences, Rostov-on-Don, (1972. -200 p). 